Commentary

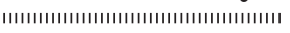

(Special Topic)

\title{
Synthesis and biological activity of novel anti-juvenile hormone agents
}

\author{
Eiichi Kuwano, * Norihiro FustTa, Kenjiro Furuta and Naotaka Yamada \\ Laboratory of Pesticide Chemistry, Department of Applied Genetics and Pest Management, Faculty of Agriculture, \\ Kyushu University, Fukuoka 812-8581, Japan
}

(Received October 17, 2007; Accepted November 5, 2007))

\begin{abstract}
Ethyl 4-[2-(tert-butylcarbonyloxy)butoxy]benzoate (ETB) is a partial juvenile hormone (JH) antagonist which targets larval epidermis. We converted ETB to ethyl 4-(2-substituted alkyloxy)benzoates. These compounds induced precocious metamorphosis in the silkworm, Bombyx mori, which is a clear sign of JH deficiency, and their activity was completely counteracted by the simultaneous application of a JH agonist, methoprene. Among these novel compounds, ethyl 4-(2-benzylhexyloxy)benzoate (KF-13) showed high precocious metamorphosis-inducing activity at low doses, but its activity decreased with increasing dose, probably due to their JH-like activity. KF-13 is an enantiomeric compound. The $(S)$-enatiomer of KF-13 was more active than the $(R)$-enantiomer at low doses, but at high doses the activity was reversed $(R>S)$. Hemolymph JH esterase activity, which is indispensable for the initiation of pupation in normal last-instar larvae, was induced in 4th-instar larvae by treatment with KF-13. 2-(6-Methyl-3-pyridyloxy)hexyl and 2-phenoxyhexyl analogs showed JH activity when topically applied to allatechtomized 4th-instar larvae. (C) Pesticide Science Society of Japan
\end{abstract}

Keywords: juvenile hormone, anti-juvenile hormone, precocious metamorphosis, juvenile hormone esterase.

\section{Introduction}

Juvenile hormone $(\mathrm{JH})$ secreted from the corpora allata, which is regulated by the brain, influences a wide range of physiological events in both developing and mature insects. ${ }^{1)}$ If the $\mathrm{JH}$ titer is high in the larval stage, larval-larval molt is induced when ecdysteroids are secreted. However, if ecdysteroids are secreted under reduced concentrations of JH (low $\mathrm{JH}$-titer) and the absence of $\mathrm{JH}$, larvae molt to pupae, which molt to adults, respectively. JH is also required in adults, for the reproductive functions of adults, such as pheromone biosynthesis, ovarian development, maturation of eggs in females, and accessory reproductive gland development in males. Diapause is also regulated by $\mathrm{JH}$ in certain insects. Therefore, compounds inducing JH deficiency are potentially useful not only as biochemical probes to assist in elucidating the role of $\mathrm{JH}$ in insect development and reproduction, but also as insect growth regulators. These compounds are categorized as anti-JH agents. ${ }^{2}$ Although precocenes, fluo-

\footnotetext{
* To whom correspondence should be addressed.

E-mail: ekuwano@agr.kyushu-u.ac.jp

(C) Pesticide Science Society of Japan
}

romevalonate, dichloroallyl hexanoate, ethyl 4-[2-(tert-butylcarbonyloxy)butoxy]benzoate (ETB, Table 1), 1,5-disubstituted imidazoles, ${ }^{3)}$ and brevioxime ${ }^{4)}$ are known as anti-JH agents, their activities were restricted to some insects species and were not sufficiently active for practical purposes.

\section{Discovery of Novel Anti-JH Agents}

ETB is known to show both JH-like action and anti-JH action for the tobacco hornworm, Manduca sexta ${ }^{5}$ and the silkworm, Bombyx mori ${ }^{6}{ }^{6}$ depending on the dose applied. Low doses of ETB induced precocious metamorphosis but, at higher doses, precocious metamorphosis-inducing activity disappeared and, instead, JH-like activity was observed. Riddiford et al. have reported that ETB acts as a partial JH antagonist at the target tissue of the larval epidermis. ${ }^{7)}$ No other anti-JH agents with such action have been found to date and the exact mode of action of ETB is still unknown.

We modified the structure of ETB and found that ethyl 4-[2(6-methyl-3-pyridyloxy)butyloxy]benzoate (1) induced precocious metamorphosis in the larvae of B. mori even at higher doses. ${ }^{8)}$ Modifications of the ethyl side chain of $\mathbf{1}$ revealed that the butyl (2) and isobutyl (3) groups were optimal for high activity. ${ }^{9)}$ The butyl side chain was fixed in the molecule, 
and a modification was made by replacing the 6-methyl-3pyridyl moiety with other aromatic rings. Among them, the phenyl analog $\mathbf{4}$ showed activity comparable to those of $\mathbf{2}$ and $\mathbf{3}$, suggesting that 6-methyl-3-pyridyl moiety is not significant for activity. ${ }^{10)}$ In this series of compounds, the introduction of a methyl or chloro substituent on the benzene ring did not increase activity. In the alkyl 4-(2-phenoxyhexyloxy)benzoate series, only methyl and ethyl esters showed precocious metamorphosis-inducing activity. Replacement of the ester group with an ethylcarbamoyl, butanoyl, nitro or a phenoxy group dramatically decreased or eliminated activity. ${ }^{11)}$ These results indicate that the methyl or ethyl ester group plays an important role in activity.

Further structure-activity relationship studies on this series of compounds indicated that ethyl 4-(2-benzylhexyloxy)benzoate (5, KF-13) and the heptyl analog 6 showed much stronger precocious metamorphosis-inducing activity than compounds 1-4 (Table 1). ${ }^{12)}$

\section{Anti-JH Activity of KF-13}

KF-13 as well as ETB induced precocious metamorphosis in the 4th larval stage of B. mori when applied to 24-hr-old 3rdinstar larvae. None of the treated 3rd-instar larvae metamorphosed into precocious pupae in the 3rd larval stage. ETB did not induce precocious metamorphosis when applied to newly molted 4th-instar larvae, while KF-13 was found to show weak precocious metamorphosis-inducing activity against 4th-instar larvae. KF-13 clearly induced precocious metamorphosis at a low dose of $1 \mu \mathrm{g}$, but its activity comparatively decreased by increasing the applied doses. The activity of KF13 could be fully counteracted by a $\mathrm{JH}$ agonist, methoprene, but not by the dietary administration of 20-hydroxyecdysone. Conversion of the 4-ethoxycarbonyl group of KF-13 to the corresponding carboxylic acid eliminated activity, indicating that the ester group itself is responsible for activity. ${ }^{12)}$

Since a chiral carbon exists in KF-13, both enantiomers of KF-13 were prepared by using chiral auxiliary oxazolidinones. The enantiometric excess of each isomer was determined to be more than $98 \%$. The $(S)$ - $(-)$-enantiomer was more active than the $(R)-(+)$-enantiomer at doses of 0.1 and $1 \mu \mathrm{g}$, but their activity was reversed at higher doses of 10 and $40 \mu \mathrm{g}$. No consistent dose-response relationship was obtained in the $(S)$-enantiomer as well as the racemate. The activity of the racemate was approximately half as much as that of the $(S)$-enantiomer at $0.1 \mu \mathrm{g}$. Thus, the active component of $\mathrm{KF}$ 13 seemed to be the $(S)$-enantiomer. ${ }^{12)}$ ETB showing anti-JH activity at low doses and $\mathrm{JH}$ activity at higher doses in $M$. sexta larvae is entirely due to the $(S)$-enantiomer, the $(R)-(+)$ isomer being completely inactive. ${ }^{13)}$

JH esterase (JHE), which hydrolyzes the methyl ester of JH into inactive $\mathrm{JH}$ acid, plays an important role as one of the major factors involved in $\mathrm{JH}$ regulation in the last instar larvae. $^{14)}$ In the silkworm, JHE activity in the hemolymph is quite low in 4th-instar larvae and increases in 5th-instar lar-
Table 1. Structues of ETB and ethy 4-(2-substituted alkyloxy)benzoates

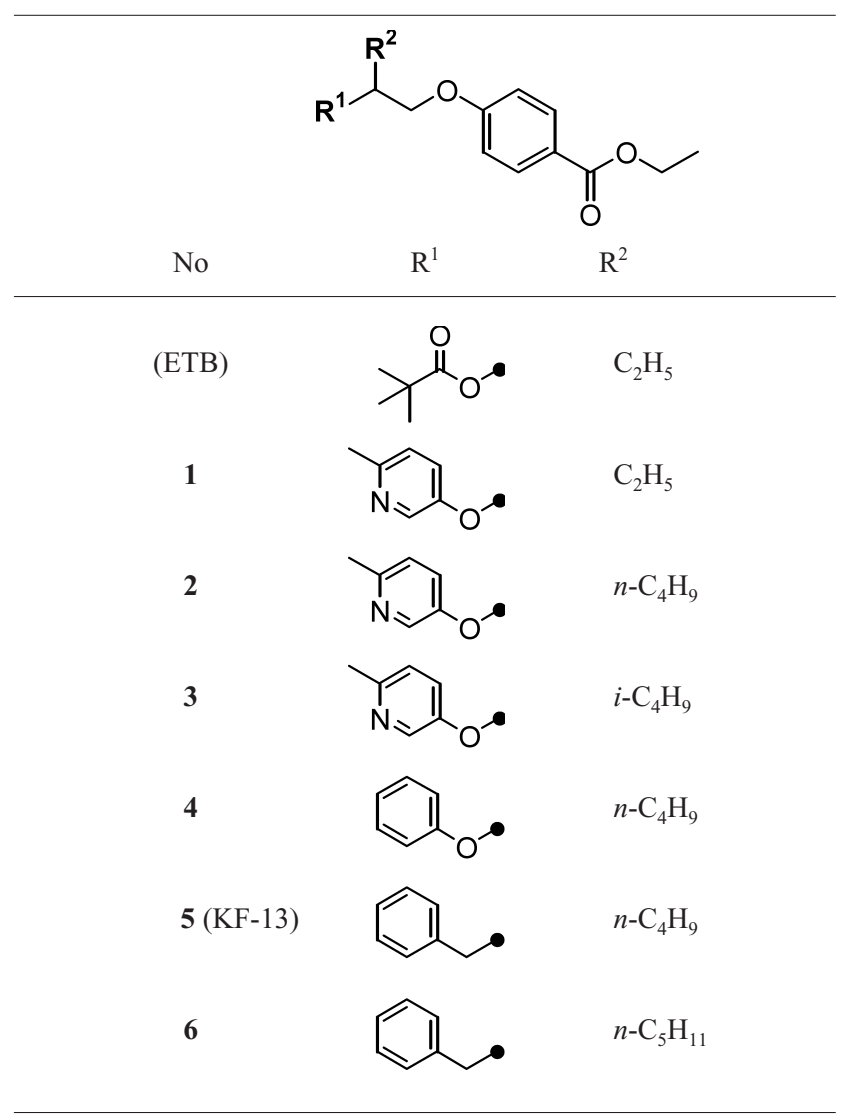

vae, thereby decreasing the $\mathrm{JH}$ titer and inducing metamorphosis. ${ }^{15)}$ We have found that compound $2^{9)}$ and KF-13 ${ }^{16)}$ induce hemolymph $\mathrm{JH}$ esterase activity during the 4th instar when applied to 3rd-instar larvae of B. mori. This result supports the concept that the appearance of JHE activity in the hemolymph is indispensable for the initiation of pupation.

\section{JH Activity of Ethyl 4-(2-Substituted hexyloxy)ben- zoates}

ETB clearly acts as a JH agonist to counteract the effect of allatectomy, the induction of precocious metamorphosis, in a dose-dependent manner. $\left.{ }^{6}\right)$ We have found that compounds 2 and $\mathbf{4}$ are effective $\mathrm{JH}$ agonists by bioassay using allatectomized 4th-instar larvae. ${ }^{17}$ ) The JH activity of $\mathbf{2}$ and $\mathbf{4}$ was less than that of ETB. Although numerous compounds such as aryl geranyl ethers, alkyl $(2 E, 4 E)$-3,7,11-trimethyl-2,4-dodecadienoates and 4-phenoxyphenoxy analogs have been found to exhibit potent $\mathrm{JH}$ activity, ${ }^{18)}$ so far no data on the anti-JH activity of these JH analogs have been presented. ETB was the only compound showing both $\mathrm{JH}$ and anti-JH activity.

It has been reported that in the early stage of the 5th-instar larvae of $B$. mori, JH titers in hemolymph decline to an undectable level, and topical application of a $\mathrm{JH}$ agonist during this period delays the initiation of larval-pupal transforma- 
tion. ${ }^{19)}$ The $(S)$-enantiomer of KF-13 as well as ETB prolonged the duration of the instar and delayed the onset of cocoon spinning when applied to 5th-instar larvae, while the $(R)$-enantiomer did not show any effect. The activity of the racemic mixture $\mathrm{KF}-13$ was less than that of $\mathrm{KF}-13(S){ }^{12)}$ These observations suggest that KF-13(S) also has JH-like activity.

\section{Conclusion}

We identified ethyl 4-(2-substituted alkyloxy)benzoates (1-6) derived from ETB as a new class of potent anti-JH agents. These compounds induced precocious metamorphosis in $B$. mori larvae, which is clearly recognized as a JH-deficiency symptom. Their activity was much higher than that of ETB. Among the compounds tested, KF-13 and $\mathbf{6}$ induced precocious metamorphosis at considerably low doses, however, their activity decreased by increasing the applied doses, probably due to their JH-like activity. Compounds $\mathbf{2}$ and $\mathbf{4}$ obviously showed $\mathrm{JH}$ activity when topically applied to allatechtomized 4th-instar larvae, but their activity was much lower than ETB.

\section{Acknowledgment}

This work was supported by a grant-in-aid to E. K. for scientific research (no. 17208007) from the Ministry of Education, Culture, Sports, Science, and Technology of Japan.

\section{References}

1) L.M. Riddiford: Adv. Insect Physiol. 24, 213-274 (1994).

2) G. B. Staal: Ann. Rev. Entomol. 31, 391-429 (1986).

3) G. C. Unnithan, J. F. Anderson, T. Hisano, E. Kuwano and R. Feyereisen: Pestic. Sci. 43, 13-19 (1995).
4) M. Castillo, P. Moya, F. Couillaud, M. D. Garcera and R. Martinez-Pardo: Arch. Insect Biochem. Physiol. 37, 287-294 (1998).

5) G. B. Staal: Entomol. Exp. Appl. 31, 15-23 (1982).

6) K. Kiguchi, T. Mori and H. Akai: J. Insect Physiol. 30, 499-506 (1984).

7) L. M. Riddiford, C. R. Roseland, S. Thalberg and A. T. Curtis: J. Insect Physiol. 29, 281-286 (1983).

8) H. Ishiguro, N. Fujita, I-H. Kim, T. Shiotsuki and E. Kuwano: Biosci. Biotechnol. Biochem. 67, 2045-2047 (2003).

9) N. Fujita, K. Furuta, H. Shirahashi, S. Hong, T. Shiotsuki and E. Kuwano: J. Pestic. Sci. 30, 192-198 (2005).

10) K. Furuta, H. Shirahashi, H. Yamashita, K. Ashibe and E. Kuwano: Biosci. Biotechnol. Biochem. 70, 746-748 (2006).

11) K. Furuta, H. Shirahashi, K. Ashibe, H. Yamashita, S. Nishikawa, N. Fujita, N. Yamada and E. Kuwano: J. Fac. Agr., Kyushu Univ. 51, 303-308 (2006).

12) K. Furuta, K. Ashibe, H. Shirahashi, N. Fujita, H. Yamashita, N. Yamada and E. Kuwano: J. Pestic. Sci. 32, 99-105 (2007).

13) D. H. S. Horn, R. H. Nearn, J. B. Siddall, G. B. Staal and D. C. Cerf: Aust. J. Chem. 36, 1409-1417 (1983).

14) T. C. Sparks, B. D. Hammock and L. M. Riddiford: Insect Biochem. 13, 529-541 (1983).

15) T. Shiotsuki, F. Yukuhiro, M. Kiuchi and E. Kuwano: J. Insect Physiol. 45, 1049-1055 (1999).

16) N. Fujita, K. Ashibe, H. Yamashita, N. Yamada, E. Kuwano and T. Shiotsuki: Abstr. 9th International Conference on Juvenile Hormones, p. 71, 2007.

17) N. Fujita, K. Ashibe, N. Yamada, T. Shiotsuki, M. Kiuchi and E. Kuwano: Biosci. Biotechnol. Biochem. 71, 2333-2334 (2007).

18) C. A. Henrick: "Agrochemicals from natural products," ed. by C. R. A. Godfrey, Marcel Dekker, New York, pp. 147-213, 1995.

19) S. Sakurai: J. Insect Physiol. 30, 657-664 (1984). 